\title{
An Experiment that Shaped the Physics of the Century
}

\section{Rohini Godbole}

In this article it is pointed out how the different layers of substructure of matter were revealed to us by experiments which were essentially very similar to the famous $\alpha$-particle scattering experiment performed by Rutherford. This experiment, which revealed the nuclear structure of an atom, paved the way towards our current understanding of the fundamental constituents of matter and shaped the course of physics for the 20th century.

\section{1. 'Looking' Inside Matter}

We are all celebrating the centenary of the remarkable discovery by Rutherford that all the positive charge and almost all the mass of an atom is concentrated in a tiny region, christened 'nucleus of an atom' by him. This discovery, in fact, shaped the course of physics for the entire century ${ }^{1}$. After the discovery of atomic structure of matter, this was the next step into our journey towards an understanding of what lies at the 'heart of matter'. Rutherford in fact, 'split' the atom! The importance of this step is underscored when we note that a physicist like Feynman had hailed our knowledge of atomic structure of matter as the one piece of understanding worthy of passing on to the future, should all but one piece of the entire scientific knowledge be destroyed. The series of experimental and theoretical investigations that began with Rutherford's experiment have now helped us understand that the basic building blocks of nature are quarks and leptons, the quarks making up protons/neutrons which in turn make up the nuclei that form then atoms along with electrons. The atoms in turn make the molecules and so on. In fact,

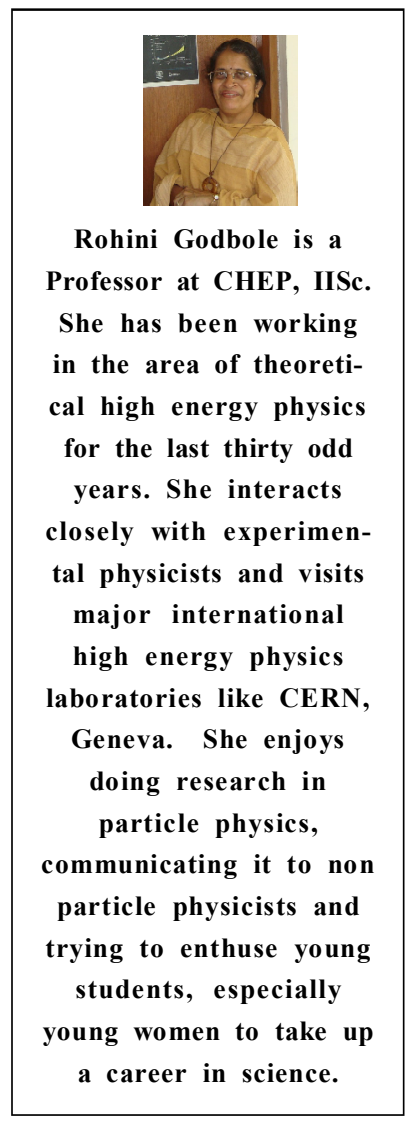

${ }^{1}$ It is interesting that Rutherford's Nobel Prize was for the 'Chemistry of Radioactive substances'! This makes Rutherford one of the two scientists, along with Albert Einstein, who did not get the Nobel Prize for their most well-known work!

\section{Keywords}

Rutherford, structure of photon, quark, deep inelastic scattering. 
Figure 1. Different layers of structures at different distance/energy scales.
The experiments being carried out today at the LHC are but a logical conclusion of the kind of experiment performed by

Rutherford.

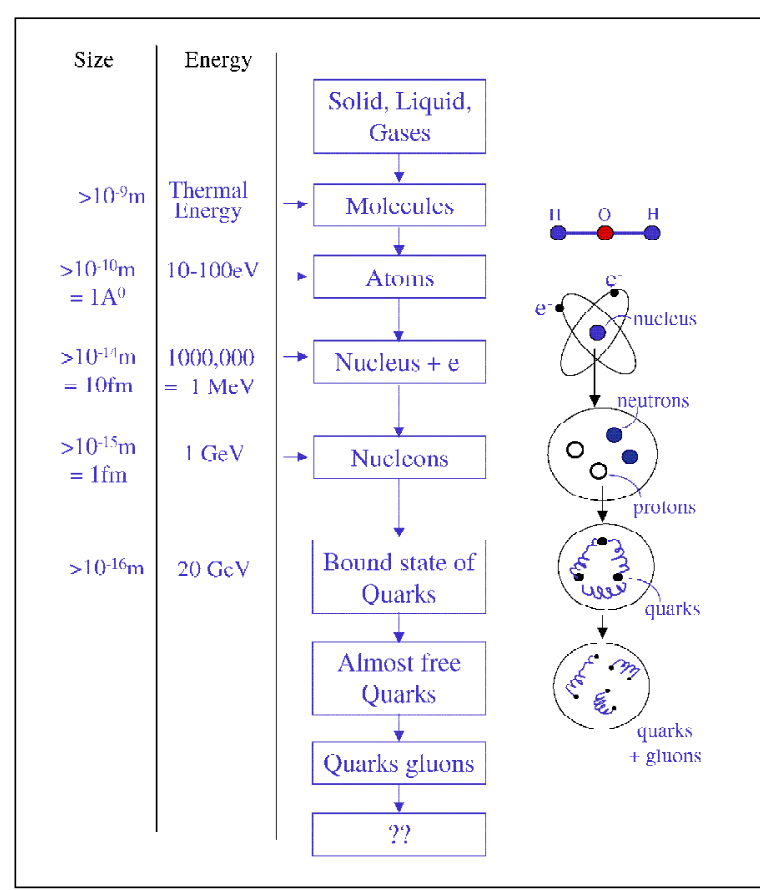

the simple drawing in Figure 1 depicts how different layers of structure of matter have been revealed at different distance/energy scales. This picture helps us appreciate the magnitude of importance of Rutherford's discovery.

In general, there have been two basic ways in which physicists have arrived at this current understanding of the substructure of matter. One is by noting similarities and patterns in the properties of the composites like atoms, nuclei and various particles such as proton, neutron, pions, etc., - and the second is to scatter off beams of particles from a target. Rutherford's experiment has pioneered the second way. In some sense the experiments being carried out today at the LHC (Large Hadron Collider) - where relativistic beams of protons or positive heavy ions collide with each other, hunting possibly for answers about the laws of physics that function at the heart of matter and at the beginning of the Universe - are but a logical conclusion of the kind of experiment performed by Rutherford. 


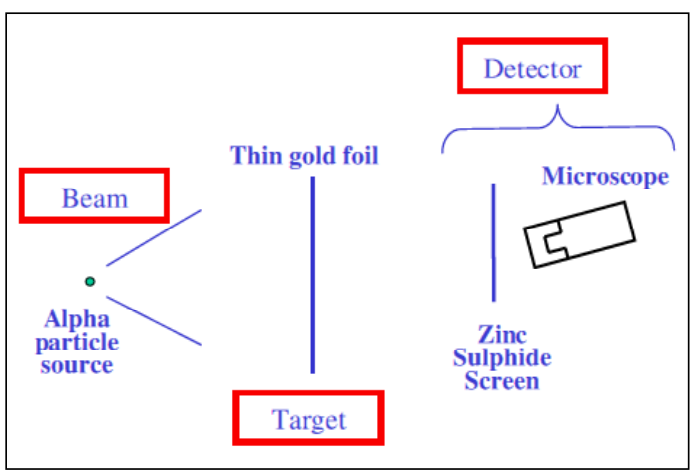

\section{Large-Angle Scattering Reveals the Atomic} Nucleus

Let me recall here the basic arrangement of Rutherford's experiment (Figure 2). He studied scattering of energetic $\alpha$ particles (the beam) emitted by radioactive nuclei from a thin gold foil (the target) and the scattered $\alpha$ particles were counted with a microscope through the scintillations these produced on hitting the zinc sulphide screen (the detector). Here the experimental observation was that the fraction of $\alpha$ particles scattered at large angles was much larger than expected if the positive charge in the atom was spread out all over the atom. In Rutherford's words, "It was about as credible as if you had fired a fifteen inch shell at a piece of tissue paper and it came back and hit you." A simple understanding as to why this indicates that the charge and mass was concentrated at a 'point' can be obtained by looking at Figure 3. As one sees from Figure 3a, the smaller the
Figure 2. A simple depiction of Rutherford's experiment.

Figure 3. A schematic depiction why substructure will lead to more large angle scattering.

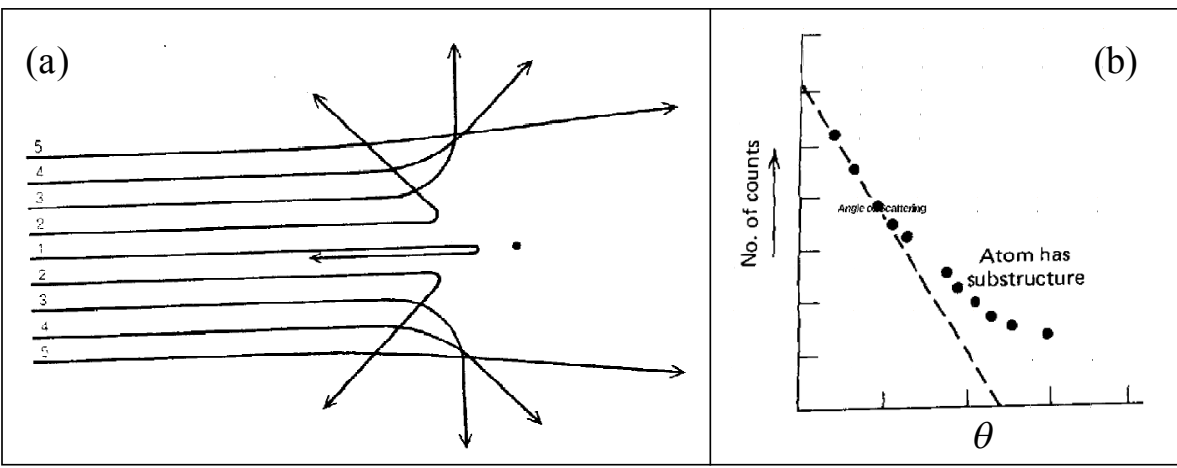




\begin{tabular}{r}
\hline The substructure \\
of an atom was \\
revealed through \\
the observed \\
angular \\
distribution of the \\
scattered $\alpha$ \\
particles.
\end{tabular}

Rutherford's

experiment showed

that the atom had a point-like nucleus; our current wisdom is that this 'point-like'

nucleus is further made up of nucleons: neutrons and protons. This has simply to do with our ability to 'resolve'. impact parameter of the approach of the $\alpha$ particles, the higher will be the angle through which it will get deflected. If the electrostatic charge felt by the incoming $\alpha$ particles is concentrated in a small region, one would expect more scattering through large angles than otherwise would be the case (Figure $3 \mathrm{~b}$ ). The $x$-axis here is the angle of scattering. This shows how the substructure of an atom was revealed through the observed angular distribution of the scattered $\alpha$ particles.

Now, the watchful among the readers may point out an obvious flaw in what I have said so far. If Rutherford's experiment showed that the atom had a pointlike nucleus, how is it that as per our current wisdom summarised in Figure 1, this 'point-like' nucleus is further made up of nucleons: neutrons and protons. This has simply to do with our ability to 'resolve'. Recall that even when we decipher the structure of an object visually, it involves scattering of light from the object which we see with our eyes and/or microscopes. In this case, smaller the wavelength of the light used, higher is the resolving power. The resolving power possible in the scattering experiments like that of Rutherford is decided by the 'wavelength' of the probing beam of particles. De Broglie's hypothesis of wave-particle duality, put forward in 1924 following Einstein's idea that light shows both wave like and particle like behaviour, implies that a beam of particles with momentum $|\vec{p}|=p$, is given by $\lambda=h / p$. Here $h$ is the Planck's constant. While for a particle moving with a non-relativistic velocity $v=|\vec{v}|$, the momentum is given by $p=m_{0} v$ for a particle of rest mass $m_{0}$, in general the magnitude of the momentum $p$ is related to the energy $E$ of the particle via $E^{2}=m_{0}^{2} c^{4}+p^{2} c^{2}$. Since the energies of the $\alpha$ particles used by Rutherford were of the order of a few million electron volts ( $1 \mathrm{eV}$ being the energy gained by an electron when it falls through a potential difference of 1 volt), his probe could resolve a size greater than 
$1 / 100$ th of an Angstrom. As we know now the size of a nucleus is a few Fermi, which is 100 thousandth of an Angstrom. Thus as far as Rutherford's experiment was concerned, the nucleus was a 'point' with an extension smaller than the least count of his probe, viz., the wavelength of the $\alpha$ particles. Thus this probe could resolve the atom with the size of few Angstroms into a point-like nucleus and electrons, but not further.

\section{Nuclear Analog of Rutherford's Experiment}

The study of properties of nuclei and observed systematics in their masses, magnetic moments, etc., had already indicated to the physicists that the nuclei too may be composites of nucleons. To determine the spatial distribution of the mass and charge of a nucleus and/or a nucleon one needs to do a nuclear analog of Rutherford's experiment, but now with beams of particles accelerated to high energies such that the corresponding wavelengths, given by de Broglie's formula above, are smaller than those of the $\alpha$ particles used by Rutherford. Again to quote Rutherford, "It has been long been my ambition to have available a copious supply of atoms and electrons which will have energies transcending those of the $\alpha, \beta$ particles." Rutherford's wish was fulfilled by Cockroft and Walton who built, in 1932, the first accelerator (and won a Nobel Prize for that in 1951!). Beginning from there, the development in accelerator physics and nuclear/particle physics went hand in hand.

In 1951, the first electron-nuclei scattering experiments were done with electron beams of about $15.7 \mathrm{MeV}$. In 1953, Hofstadter performed the nuclear analog of Rutherford's experiment with still higher energy electron beams, accelerated to energies of a few hundred $\mathrm{MeV}$. These electrons had wavelengths substantially smaller than those of the $7 \mathrm{MeV} \alpha$ particles used by Rutherford.
"It has been long been my ambition to haveavailablea copious supply of atoms and electrons which will have energies transcending those of the $\alpha, \beta$ particles." - Rutherford

\author{
Rutherford's wish \\ was fulfilled by \\ Cockroft and \\ Walton who built, \\ in 1932, the first \\ accelerator.
}


Figure 4. The schematic of the nuclear analog of the Rutherford experiment performed at the Standford Linear Accelerator (SLAC).

${ }^{2}$ You can get the information on the actual 2-mile long accelerator which was built at Stanford and the detector which was used to detect and measure properties of the scattered electron from the website of the SLAC laboratory:

http://www2.slac.stanford.edu /vvc/nobel/1990nobel.html, or Interactions.org or http://www.physics.ox.ac.uk/ documents/PUS/dis/SLAC.htm

Figure 5. The panel on the left shows the Standard Linear Accelerator and the one at right shows the detector. Courtesy: SLAC

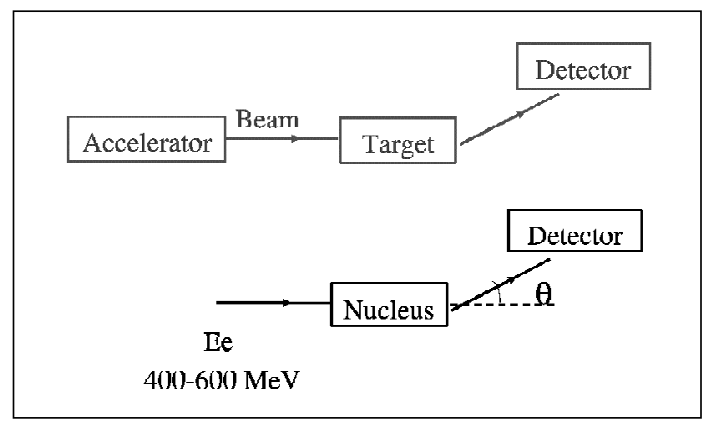

Note the similarity between the beam-target-detector arrangement in Figure 2 and that in Figure $4^{2}$. The process studied by Hofstadter was

$$
e^{-}\left(E_{\mathrm{e}}\right)+A \rightarrow e^{-}\left(E_{\mathrm{e}}^{\prime}\right)+A .
$$

In Rutherford's experiment the detector could be moved easily to measure $\alpha$ particles scattered at different angles. In case of Hofstadter's experiment the huge detector built for the purpose, could catch the scattered electrons at a few fixed angles. Once the angle of the scattered electron was given, the energy $E_{\mathrm{e}}^{\prime}$ was uniquely fixed. It is not too difficult to calculate the fractional number of electrons which would be scattered through a solid angle $\mathrm{d} \Omega . \mathrm{d} \sigma / \mathrm{d} \Omega$ is proportional to this fraction. It is possible to show that, for a spherically symmetric
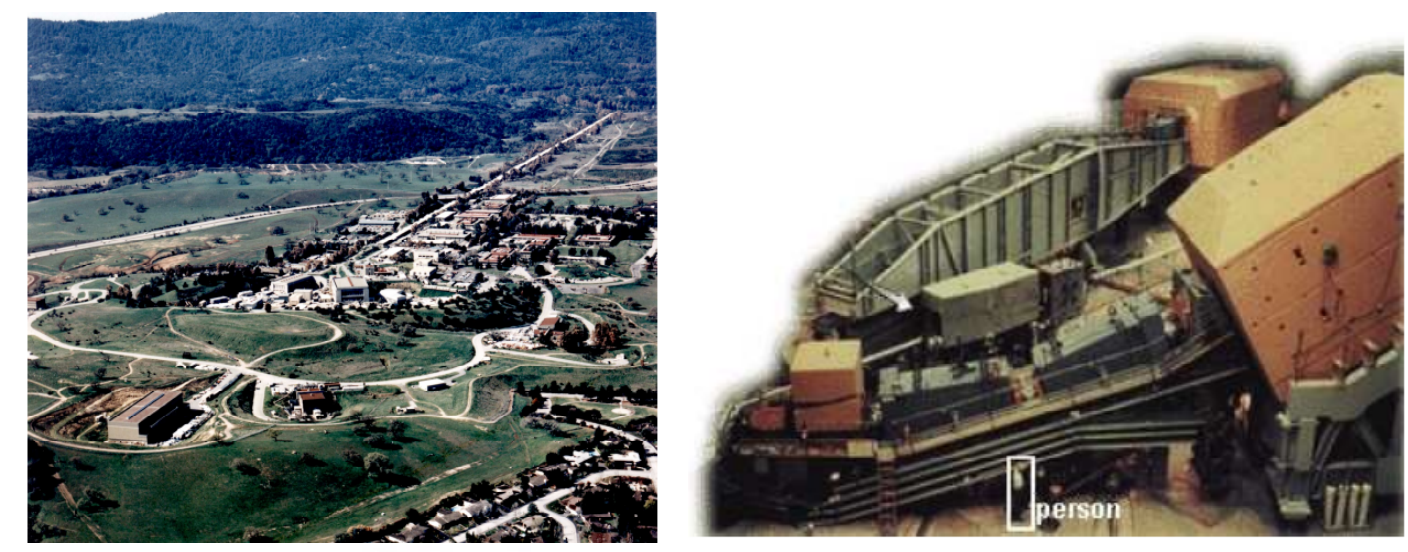
charge distribution,

$$
\left(\frac{\mathrm{d} \sigma}{\mathrm{d} \Omega}\right)_{\text {charge distn. }}=\left|F\left(Q^{2}\right)\right|^{2}\left(\frac{\mathrm{d} \sigma}{\mathrm{d} \Omega}\right)_{\text {point }},
$$

where $F\left(Q^{2}\right)$ is nothing but the Fourier transform of the 'normalised' charged distribution $\rho(\vec{R})$, with $Q^{2}=|\vec{q}|^{2}$. $\vec{q}$ is the momentum transfer from the incoming electron to the scattered electron, given by $\overrightarrow{p_{\mathrm{e}}}-\overrightarrow{p_{\mathrm{e}}^{\prime}}$. $Q^{2}$ is a more convenient variable than the scattering angle and is clearly related to it. The distribution of the scattered electron at different angles, which in turn gave $F\left(Q^{2}\right)$, indicated that the charge of the nucleus was concentrated in a region of size of the order of a few Fermi's (1 Fermi $=100000$ th of an Angstrom). Just like before, the information on electrons scattered at different angles indicated the presence of a mass/charge distribution and gave an idea of its extension. In principle, similar measurements can also yield information on the 'shapes' of nuclei as well.

To summarise, the spatial distribution of the target modifies the $Q^{2}$ dependence compared to the expectation for a point scatterer. For a point scatterer, by definition $F\left(Q^{2}\right)$ will be a constant. In fact it can be shown ${ }^{3}$ that at $Q^{2} \ll 1 /<R^{2}>, F\left(Q^{2}\right)=1-<R^{2}>Q^{2} / 6$. This formula then very clearly explains why Rutherford 'saw' the nucleus to be a 'point' even though now we know it to be as big as a few Fermi's. Our ability to infer and study the structure of an object from scattering experiments is possible only when $\left\langle R^{2}>Q^{2} \simeq 1\right.$. That is, smaller the spatial extension, higher the energy required to see the evidence for this structure. Hence, for energies such that $\lambda_{\mathrm{e}} \sim R_{\text {target }}$, one sees a deviation of $F\left(Q^{2}\right)$ from $\sim 1$. This is exactly how one got information on the spatial extension of the nucleus.
3 Incidentally, this way of obtaining information about mass/ charge distributions of the target is very similar to how one obtains the crystal structure by looking at the X-ray diffraction patterns for solids. 
Hofstadter performed the proton/neutron analog of the Rutherford experimentin

1954.

\section{Proton Too has Finite Size as well as Con- stituents!}

Since the nucleus was expected to be a composite of nucleons the finite size of the nucleus did not come as a surprise to anybody. The next step was to perform the analog of Rutherford experiment but with a proton target; the nucleus was replaced by a proton, and electron beam energies were raised to $2000 \mathrm{MeV}$ and above (Figure 4). It is interesting to note that physicists had reasons to expect that the proton too may be a composite. The value of 5.58 found experimentally for its gyromagnetic ratio was very different from the value 2 of an electron. It was known that for any spin-half charged point particle the expected value for the gyromagnetic ratio is 2 according to the Dirac equation, which is nothing but a relativistic wave equation for a spin-half particle. Worse still, neutron which is neutral should have no magnetic moment at all, but in reality has magnetic moment $=$ $-1.91 \frac{|e|}{2 M_{\mathrm{p}}}$. This indicated that the proton and neutron must be at least charge distributions.

Hofstadter performed the proton/neutron analog of the Rutherford experiment in 1954. The exact process studied by him was:

$$
e\left(E_{\mathrm{e}}\right)+p \rightarrow e\left(E_{\mathrm{e}}^{\prime}\right)+p .
$$

Energy and momentum conservation suggests that for a given value of incident electron energy $E_{\mathrm{e}}$ and scattering angle $\theta$, the scattered electron can only have a fixed value of energy $E_{\mathrm{e}}^{\prime}$ given by

$$
E_{0}=\frac{E_{\mathrm{e}}}{\left(1+2 E_{\mathrm{e}} / M_{\mathrm{p}} \sin ^{2}(\theta / 2)\right)} .
$$

The finite size of the proton was confirmed by these scattering experiments (just like that for nuclei) from measurements of the form factors $F\left(Q^{2}\right)$ for protons/neutrons and studying their $Q^{2}$ dependence. The experimental 
results indicated that the size of the proton $\sim 100,000$ times smaller than an atom: a Fermi. Hofstadter was awarded the Nobel Prize in Physics for the year 1961 for this research into sizes of nuclei and nucleons.

A natural question to ask then was: Is this proton just a charge distribution OR is there something inside? The real surprise came when $E_{\mathrm{e}}$ was increased even further! The process studied now was slightly different from the good old Rutherford scattering:

$$
e^{-}+p \rightarrow e^{-}+X,
$$

where $\mathrm{X}=\pi, K, p, \bar{p} \ldots$; and we sum over all $\mathrm{X}$. Such a measurement is called inclusive measurement. Since the final state now contains more particles than the proton, this process is called Deep Inelastic Scattering (DIS). The energies of the electron $E_{\mathrm{e}}$ were now increased yet again by a factor of 5-10 more, to 10,000-20,000 million electron volts (10-20 Giga electron Volts: GeV). The resolution possible with this electron beam was now $1 / 100$ compared to the size of the $p / n$. So this should be able to 'see' inside the proton and find out whether it too was made up of something more fundamental.

The surprising fact was that unlike the elastic scattering process of equation (1), the energy of the scattered electron $E_{\mathrm{e}^{\prime}}$, for a given angle of scattering, was found to have many different values and not just one single value $E_{0}$. This led the physicists to suspect that, maybe, the $p$ had something inside it. At still higher values of $E_{\mathrm{e}}$, the scattered electron again began to have a unique value $E_{0}^{\prime}$, different from that for a proton $E_{0}$, given by equation (2). This observation in the experiment performed by Friedman, Kendall and Taylor in 1968 could be interpreted, through the insight offered by Feynman and Bjorken, to mean that at these high energies the $\lambda_{\mathrm{e}}$ was now small enough to feel the individual scatterers inside the proton ${ }^{4}$. The experimentalists were awarded the Nobel Prize in the year 1990. The main point here
Is the proton just a charge distribution

$\mathrm{OR}$ is there something inside?
${ }^{4}$ It is another very interesting story that similar experiments, at still higher energies, could be used to show that these scatterers with an electromagnetic charge inside the proton, seemed to have exactly the same properties as the quarks postulated by Murray Gell-Mann and the neutral scatterers could be identified with the gluons required in a theory of strong interactions put forward by Gell-Mann, Fritsch and Leutwyler. But that is a story for another day. 


\begin{tabular}{r|}
\hline The methodology of \\
the Rutherford \\
experiment \\
continues to guide \\
us even today!
\end{tabular}

${ }^{5}$ Compare this with the energy of $10-20 \mathrm{GeV}$ of the first electron beams at SLAC, which discovered that proton has quarks inside it.

Address for Correspondence Rohini Godbole Centre for High Energy Physics Indian Institute of Science Bangalore 560 012, India. Email: rohini@cts.iisc.ernet.in was again that the experiments indicated the presence of scattering at larger angles than expected if the proton did not have a structure, just like Rutherford's experiment.

The above-mentioned experiments on electron-proton scattering at the Stanford Linear Accelerator (SLAC) were followed, between 1970-1990, with those using $\mu$ (muon) and $\nu$ (neutrino) beams of energies upto 800 $\mathrm{GeV}$ incident on nuclei and protons. The last word in probing the structure of the proton by colliding it with an electron beam was given by the experiments at the ep collider HERA at the German high energy physics laboratory DESY in Hamburg, which collided $e^{-} / e^{+}$ beams of $30 \mathrm{GeV}$ on proton beams of $800 \mathrm{GeV}$. This corresponds to doing an experiment with electron beams of energy $\sim 10^{5} \mathrm{GeV}$ incident on a stationary proton target ${ }^{5}$. The corresponding wavelengths of the electron beam are then of the order of $10^{-18} \mathrm{~m}=10^{-5} \mathrm{fm}$. These experiments have not revealed evidence for any further substructure. Not just that, the predictions for a very wide class of processes from theories which assume the quarks to be absolutely point-like upto very high energies seem to agree with experimental measurements to a very high degree of accuracy. Thus we have an 'indirect' indication that the quarks do not seem to have a substructure. It is a matter of great interest that even now, one of the first experimental results at the LHC, was about putting limits on the substructure of quarks $[1,2]$, by once again looking whether we have an excess of scattering at higher angles than expected from our theories. So the methodology of the Rutherford experiment continues to guide us even today!

\section{Suggested Reading}

[1] S Chatrchyan et al, [CMS Collaboration], Phys. Lett. Vol.B704, p.123, 2011; [arXiv:1107.4771 [hep-ex]].

[2] G Aad et al, [ATLAS Collaboration], [arXiv:1108.6311 [hep-ex]]. 